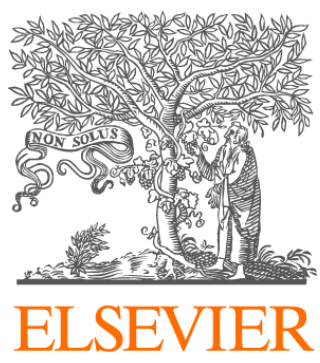

Since January 2020 Elsevier has created a COVID-19 resource centre with free information in English and Mandarin on the novel coronavirus COVID-

19. The COVID-19 resource centre is hosted on Elsevier Connect, the company's public news and information website.

Elsevier hereby grants permission to make all its COVID-19-related research that is available on the COVID-19 resource centre - including this research content - immediately available in PubMed Central and other publicly funded repositories, such as the WHO COVID database with rights for unrestricted research re-use and analyses in any form or by any means with acknowledgement of the original source. These permissions are granted for free by Elsevier for as long as the COVID-19 resource centre remains active. 


\title{
INTERNATIONAL EMS
}

\section{New Paradigm for Protection:}

\section{The Emergency Ambulance Services in the Time of Severe Acute Respiratory Syndrome}

\author{
Fatimah Lateef, MBBS, Swee Han Lim, MBBS, Eng Hoe Tan, MBBS, MSc (PH)
}

\begin{abstract}
Severe acute respiratory syndrome (SARS) is a newly emerging and highly infectious form of atypical pneumonia with a high rate of transmission, especially among health care workers. With SARS, certain policies had to be implemented rapidly by the emergency ambulance services and the Ministry of Health to support and protect all personnel adequately. The authors discuss the changes in policies and personnel behavior, the training and education that had to be disseminated widely, and certain alternatives in policies such as transportation. The authors hope to share their experience in the implementation of these strategies by the Singapore Civil Defence Force and stress the importance of the psychological preparedness of the paramedics and prehospital care providers worldwide in this era of SARS. Key words: severe acute respiratory syndrome; paramedics; emergency ambulance services; personal protective equipment.
\end{abstract}

\section{PREHOSPITAL EMERGENCY CARE 2004:8:304-307}

Singapore is a cosmopolitan city at the crossroads of Asia, with a land area of $644 \mathrm{~km}^{2}$ and a population of 4 million. The emergency ambulance service (EAS) is run by the Singapore Civil Defence Force (SCDF) and has a fleet of 30 ambulances. It is a single-tier system, which serves the whole country and handles approximately 75,000 calls annually. ${ }^{1,2}$ The paramedics on the EAS perform basic life support skills and are trained to defibrillate with the use of automated external defibrillators (AEDs). They also start intravenous lines

Received July 10, 2003, from the Department of Emergency Medicine (FL, SHL), Singapore General Hospital; and Singapore Civil Defence Force (EHT), Singapore. Revision received October 20, 2003; accepted for publication October 23, 2003.

Address correspondence and reprint requests to: Fatimah Lateef, MBBS, Consultant, Department of Emergency Medicine, Singapore General Hospital, Outram Road, Singapore 169608. e-mail: <gaefal@ sgh.com.sg > or <f_lateef@hotmail.com>

doi:10.1016/j.prehos.2003.12.016 and administer certain drugs (e.g., adrenaline, dextrose). The complete ambulance crew comprises of a driver (trained in first aid and basic life support), a qualified paramedic, and a paramedic assistant who is a trained medical orderly. In response to road traffic accidents and certain medical emergencies such as cardiac arrest, a first line of response is dispatched before the ambulances. This represents the fast response paramedics (FRPs). They are paramedics with the same qualifications as the ambulance paramedics but they have in addition to this, training in riding motorcycles, which includes defensive riding. They carry an AED as well as essential first-response equipment on their motorcycles. These FRPs work alone and will usually hand over the cases they have attended to the ambulance paramedics for transport and further management. ${ }^{1}$

Severe acute respiratory syndrome (SARS) is a form of atypical pneumonia, which was first seen in November 2002 in the Guangdong Province of China. It is a newly emerging and highly infectious disease with a high rate of transmission, especially among health care workers. As the world has become a "global village," SARS has become a problem of the "village." The SARS outbreak in Singapore begun in March 2003 and was traced to a traveler returning from Hong Kong. ${ }^{3}$

Since then, several index cases and their clusters have been identified and traced. The social and economic impact of SARS has been tremendous. Up to June 20, 2003, there have been a total of 206 cases and 32 deaths (15.5\%) in Singapore.

On March 12, 2003, the World Heath Organization (WHO) issued a global alert on SARS. WHO defines a suspected case of SARS as one with documented fever (temperature $\geq 38^{\circ} \mathrm{C}$ ), lower respiratory tract symptoms, and contact with a person who has SARS with a history of traveling to an area of documented transmission. A probable case of SARS, on the other hand, is similar to a suspected case but has chest x-ray 
findings of pneumonia, acute respiratory distress syndrome (ARDS), or an unexplained respiratory illness resulting in death with autopsy findings of ARDS without any other identifiable causes. ${ }^{4,5}$

\section{Changed Practices}

With the outbreak of SARS, the EAS, an integral component of the overall health care system, had to implement certain practices rapidly. The coordinated efforts of the SCDF with the Ministry of Health were extremely important in this respect. Mandatory steps from the preparatory phase to the initial point of contact and subsequent disposition of patients were highlighted and taught to all personnel. The best strategy was to have a high index of suspicion and be vigilant at all times for every patient. Prevention is best in the management of any infectious disease and, hence, for SARS, it behooves the system to prevent its spread. Because it would take up to one week before a diagnosis of SARS could be confirmed, universal precautions had to be undertaken for every patient being treated. This entails the adoption of full protection for every ambulance crewmember. To date, a total of seven probable and five suspected cases were conveyed by the EAS out of a total of 204 patients with SARS-like signs and symptoms.

\section{Change in Personnel Behavior}

It was compulsory for all ambulance crewmembers to use personal protective equipment (impervious gowns over their uniforms, N95 masks, ${ }^{*}$ eye goggles, head covers, and disposable gloves) when attending to all cases. The gowns and gloves were changed after every case. The impervious gowns served as protective apparel, which provided a barrier to prevent contamination of the uniform and skin. Goggles, or face shields, helped to avoid splashes or sprays of body fluids or secretions to the mucous membranes of the eyes. The gloves provided a protective barrier and prevented gross contamination of the hands, reducing the likelihood of spread of microorganisms from person to person or from fomites (surface or equipment) to person.

Hand hygiene was also stressed to all. Because SARS appears to be spread by direct contact with respiratory secretions, touching of contaminated objects was

\footnotetext{
${ }^{*}$ N95 masks: Health care particulate respirator and surgical masks designed to provide protection for the user. There is filter efficiency level of 95\% or greater against particulate aerosols free of oil. They are fluid-resistant and disposable and satisfy the Centers for Disease Control and Prevention criteria for Mycobacterium tuberculosis exposure control. They reduce the exposure to airborne particles ranging from 0.1 to $>10.0 \mu \mathrm{m}$. N95 masks provide $>99 \%$ bacterial filtration efficacy (BFE) against user-generated microorganisms.
}

a potential source of transmission. Hand hygiene (either by handwashing or the use of hand rub solution) was critical before and after contact with every patient. Once worn in the presence of a patient who was suspected of having SARS, the mask was considered potentially contaminated with infectious material (even if not visible), and touching of the mask was avoided. Reusable equipment such as stethoscopes was disinfected with alcohol-impregnated wipes after every patient.

Special disposable mechanical filters were fitted for the bag-valve-mask and suction units to filter expired air from possible patients suspected of having SARS. Because nebulization for severe asthmatics was not allowed to prevent reaerosolization of any organism, spacers for metered-dose inhalers (MDIs) had to be used.

Surveillance of all the EAS personnel was also carried out. Temperature checks were done regularly three to four times a day, even for those on their off-duty days. Those who had contact or had transported any suspected SARS or high-risk cases were also monitored closely. They had to report their temperatures and any SARS-like symptoms to a central monitoring station in the Medical Department of SCDF. Contingency plans were also in place to isolate the contacts of these ambulance crews should they be considered SARS suspects.

\section{Training and Education}

With the SARS outbreak, infection control training had to be stepped up immediately. The training sessions were carried out using a variety of teaching methods such as small-group classroom teaching, demonstration sessions, use of videotapes to highlight techniques (such as the donning of personal protective gear and effective handwashing), and hands-on practical sessions for mask fitting. Mask-fitting sessions had to be carried out for all staff to ascertain the best-sized mask for each. Hand hygiene practical sessions were supervised to ensure it was performed correctly. The infection control officer gave briefings to everyone and all this had to be carried out rapidly, over a short period, to ensure all personnel had the necessary skills and knowledge early.

Those who failed to comply were given warnings and counseled accordingly. Their conduct and practices were then monitored by their supervisor. The compliance rate was observed to be high among all staff. The rate was not objectively measured or analyzed but feedback was obtained from training supervisors, senior paramedics from the Medical Department at SCDF, and also from staff of the emergency departments who were in contact with these paramedics. 
There were also frequent updates on the SARS situation locally, as well as in the region. Updates from the hospitals were also given on some of the cases transported and suspected to be SARS. This was part of the hospital contact tracing procedure for every suspected or probable SARS case. SARS was also highlighted during the continuing education sessions for the paramedics, where the clinical presentations, the rapidly changing epidemiology, definitions, and risk factors were discussed. The history taking by paramedics now had to incorporate certain questions pertaining to risk for SARS, overseas travel, and fever. This was also performed by the emergency medical dispatcher (EMD) whenever possible. The reason for this was because the high-risk status could be identified early. Because the initial clinical features of SARS were not specific and physical signs on chest examination were minimal, they were cautioned that every patient could be a potential suspected SARS case.

The paramedics also helped to educate the public by imparting relevant and accurate information whenever the opportunity was present because, with any public health outbreak such as this, there was bound to be misconceptions with some members of the public. They also helped to stress the importance of making an honest declaration when completing the SARS health questionnaires.

\section{Transportation}

With the declaration of the SARS outbreak in Singapore, the Ministry of Health designated Tan Tock Seng Hospital (TTSH), one of the public hospitals, as the "SARS hospital." All suspected and probable cases were sent and managed there. With this, TTSH closed its emergency department to all other cases, and cases conveyed by the EAS had to be diverted to the other five public hospitals. The patient load at these other hospitals thus increased.

During this period, security at all hospital entrances was stepped up to ensure no lapses in patient screening and entry into health care institutions. If patients "walked in" to the emergency department at TTSH, they would be screened, assessed, and the appropriate advice/treatment would be given. If they required admission, they would be transferred to one of the other hospitals for further management.

The paramedics had to bear this diversion rule in mind when they made decisions about transporting patients to the nearest or most appropriate hospital. Based on history and rapid examination, if there were any suspected cases of SARS, these patients were sent to TTSH. The rule of having only one relative accompany the patient in the ambulance was discontinued except for pediatric patients. If so, the relative was to be seated in the front of the ambulance and seatbelted.

When transporting any patient, the windows of the ambulance should be kept open with the air conditioner off. This helped with ventilation within the ambulance cabin. Preventive measures were taken to reduce the heat load on the crew with their full protective clothing. This including wearing of T-shirts instead of uniforms and ensuring adequate fluid intake. These practices were essential with the hot and humid climate in Singapore.

After the patient had been sent to the receiving hospital, the paramedics had to clean all equipment and trolleys in the ambulance with antiseptic wipes and mop the floor of the ambulance with antiseptic solution. The vehicle was then aired, with all its windows and doors opened, for 30 minutes. On arrival at the hospitals, the paramedics had to comply with temperature checks and health declaration for themselves before being allowed entry into the emergency departments.

When there were uncertainties about the history, especially of a possible SARS contact, or which hospital to send the patient to, paramedics could clarify with the personnel at the control center in SCDF or the emergency physician at the receiving hospital. Checks were also made with the SARS hotline set up by the Ministry of Health through the control room, which had access to patients who were on home quarantine orders (HQO).

During this outbreak, a separate dedicated SARS ambulance service was set up to transport all nonemergency suspected and probable cases to TTSH. This was a free service with a simple single access number, 993. However, patients, irrespective of whether they were suspected to have SARS or not, continued to be treated and conveyed by the EAS when they developed any emergency medical conditions. This "ring fence" strategy was in place to reduce the likelihood of cross-infection, and it also depended on vigilant screening of all potential SARS cases by primary care physicians, paramedics, and emergency department staff. The patients on HQO also used the SARS ambulance service when they had to seek medical attention for any reason. These people had been advised not to use public transportation or to drive themselves to the hospitals to reduce any chance of infecting others.

\section{Conclusions}

With the mandatory practices implemented by SCDF, there was high compliance among the paramedics and EAS staff. As a result, there were no EAS personnel 
who were affected by SARS during the outbreak. In a situation such as this, it is important to put forth sufficient information to gain support and allay fears of the staff. These health care workers at the frontline also have concerns about spreading any likely infection to their families and loved ones.

SARS is a newly emerging infectious disease, but there are a whole range of other diseases such as tuberculosis, HIV/AIDS, and meningitis that frontline health care workers need to be aware of. EMS staff should continually be updated on relevant information pertaining to these. 6,7 The communicative strategy of the SCDF is commendable, and it helps that the paramedics were psychologically prepared for the risks ahead. They also know that they have the support of their organization in ensuring they have been given the best protection available.

\section{References}

1. Lateef F, Anantharaman V. Bystander CPR in prehospital cardiac arrest in Singapore. Prehosp Emerg Care. 2001;5:387-90.

2. Health Facts Singapore. Singapore: Ministry of Health, 2001.

3. Hsu LY, Lee CC, Green JA, et al. Severe acute respiratory syndrome (SARS) in Singapore: clinical features of index patient and initial contacts. Emerg Infect Dis. 2003;9:713-7. Also available at: http:/ / www.cdc.gov/incidod/EID/vol9no6/03-0264.htm. Accessed Jun 30, 2003

4. Centers for Disease Control and Prevention. Outbreak of SARS worldwide, 2003. MMWR. 2003;52:226-8.

5. World Health Organization. WHO Issues Emergency Travel Advisory: SARS Spread Worldwide. Available at: http://www. who.int/mediacenter/releases/2003/pr23/en. Accessed Jun 30, 2003.

6. Mencl F, Birkle M, Blanda $\mathrm{M}$, et al. EMTs' knowledge regarding transmission of infectious diseases. Prehosp Emerg Care. 2000;4:57-61.

7. Reed E, Daya MR, Jui J, et al. Occupational infectious disease exposure in EMS personnel. J Emerg Med. 1993;11:9-16.

\section{Instructions for Authors of Case Conferences}

The case conference format may be used for the presentation of interesting or unusual EMS encounters. This format can illustrate specific medical entities, unusual approaches to field management, or complex administrative issues that a field scenario may present. Authors should pay particular attention to the educational value of the manuscript, and avoid a purely descriptive approach. Features such as a team approach and innovative solutions to atypical problems should be stressed. While an abstract and specific section headings are not required, the following sections should be considered:

1. overall description of the scene, types of responding agencies and personnel, etc.

2. specific challenges encountered

3. solutions developed to address the challenges

4. discussion of medical issues involved, with review of the literature where appropriate

5. discussion of logistic and administrative issues

Title page, group authorship and acknowledgments page, references, and tables and figures (where appropriate) should follow the same format as for general manuscripts (see the "Manuscript Preparation" section of the "Instructions for Authors" following the text of most issues of Prehospital Emergency Care). 